OPEN ACCESS

Edited by:

Sukanya Narasimhan,

Yale University,

United States

Reviewed by:

Jan Perner,

Academy of Sciences of the Czech

Republic (ASCR), Czechia

Shahid Karim,

University of Southern Mississippi,

United States

*Correspondence:

Monika Gulia-Nuss

mgulianuss@unr.edu

Specialty section:

This article was submitted to

Parasite and Host,

a section of the journal

Frontiers in Cellular and Infection

Microbiology

Received: 08 March 2021

Accepted: 16 April 2021

Published: 10 May 2021

Citation:

Nuss A, Sharma A and Gulia-Nuss $M$ (2021) Genetic Manipulation of Ticks:

A Paradigm Shift in Tick and

Tick-Borne Diseases Research.

Front. Cell. Infect. Microbiol. 11:678037.

doi: 10.3389/fcimb.2021.678037

\section{Genetic Manipulation of Ticks: A Paradigm Shift in Tick and Tick-Borne Diseases Research}

\author{
Andrew Nuss ${ }^{1,2}$, Arvind Sharma ${ }^{1}$ and Monika Gulia-Nuss ${ }^{1 *}$ \\ ${ }^{1}$ Department of Biochemistry and Molecular Biology, The University of Nevada, Reno, NV, United States, ${ }^{2}$ Department of \\ Agriculture, Veterinary, and Rangeland Sciences, The University of Nevada, Reno, NV, United States
}

Ticks are obligate hematophagous arthropods that are distributed worldwide and are one of the most important vectors of pathogens affecting humans and animals. Despite the growing burden of tick-borne diseases, research on ticks has lagged behind other arthropod vectors, such as mosquitoes. This is largely because of challenges in applying functional genomics and genetic tools to the idiosyncrasies unique to tick biology, particularly techniques for stable genetic transformations. CRISPR-Cas9 is transforming non-model organism research; however, successful germline editing has yet to be accomplished in ticks. Here, we review the ancillary methods needed for transgenic tick development and the use of CRISPR/Cas9, the most promising geneediting approach, for tick genetic transformation.

Keywords: ticks, Ixodes scapularis, gene-editing, embryo-injection, CRISPR/Cas-9

\section{INTRODUCTION}

Ticks and tick-borne diseases affect animal and human health worldwide and are the cause of significant economic losses. For instance, Lyme disease alone costs approximately $\$ 1.3$ billion each year in direct medical costs in the United States (Tick-borne Disease Working Group). The tick life cycle begins with an egg containing the developing embryo that hatches into a larva. As the tick proceeds through larval and nymphal stages, a single blood meal is required at each stage, and a final large blood meal by the adult female is needed to develop an egg mass to complete the cycle. This life cycle differs vastly from hematophagous insects, where usually only the adults-and often only females-feed on vertebrate blood, and therefore only adults can vector diseases from infected animals. In contrast, ticks are obligate blood-feeders at all stages of their life cycle, making them viable to transmit pathogens at various life stages. Ticks can transmit many pathogens: bacteria, viruses, protozoans, and fungi (Jongejan and Uilenberg, 2004; Rochlin and Toledo, 2020). Borrelia spirochetes, the causative agents of Lyme disease, are among the most important pathogens transmitted by Ixodes ticks. However, several other tick-transmitted pathogens are of importance to human and animal health (Eisen and Eisen, 2018). Furthermore, as the tick feeds for extended periods (3-10 days), it interacts with its vertebrate host and can suppress the host's immune system. In addition to being a vector for pathogens, ticks can cause significant harm to their host due to feeding for a prolonged time: exsanguination when tick infestation is high, secondary infection at the bite site (Eisen and Eisen, 2018), tick paralysis when feeding occurs near the spinal cord (Pienaar et al., 2018), and reactions to tick bites such as alpha-gal syndrome (Commins and Platts-Mills, 2013; 
Steinke et al., 2015) which induces an allergy to red meat. Understanding tick biology, therefore, is an important research focus.

The process for acaricide discovery and drug/vaccine development against ticks or tick-borne pathogens heavily relies on correlating tick genotype to phenotype. To study the connection of genotype to phenotype, it is important to disrupt gene function and analyze phenotypic effect. Researchers can experimentally regulate gene expression and investigate gene function either at the translational level or at the genetic level using two main biological tools: RNA interference (RNAi) and gene knockouts using editing methods, respectively. CRISPR/ Cas9 has emerged as the most straightforward gene editing technology, in comparison to other techniques such as TALENs and ZFNs etc. because of the lower cost and ease of use. Current functional genomics research in ticks primarily depends on RNAi for gene knockdown studies (Karim et al., 2008). However, RNAi has limitations such as incomplete silencing that can vary by target gene, developmental stage, or tissue. In addition, temporary (transient) transcript knockdown limits many studies to a narrow assay window and currently puts transstadial pathogen transmission studies out of reach. Furthermore, RNAi is not suitable for overexpression and transcript rescue experiments required to study functions of the genes that are downregulated, for instance due to pathogen infection. Moreover, RNAi is mostly applicable to adult and sometimes nymphal stages, making gene function studies in embryos and larvae challenging. Still, the reversible nature of knockdowns by RNAi makes it possible to verify the phenotypic effect by restoring protein expression to normal in the same cells. In addition, it may permit knockdown of genes in later life stages that otherwise would result in irreversible developmental abnormalities if knocked down in earlier life stages.

In contrast to RNAi, the advantages of genomic knockouts are absolute silencing, completely blocking protein expression, eliminating any confounding effects from remnant low levels of protein expression post knockdown. Targeted gene knockout and knock-in approaches are therefore highly desirable to investigate tick gene functions that are unanswerable by RNAi alone. The CRISPR/Cas genetic manipulation system is revolutionizing the field of biology, including entomology, by enabling the genetic transformation of diverse arthropods (Bassett and Liu, 2014; Ma et al., 2014; Gilles et al., 2015; Kohno et al., 2016; Li et al., 2017b; Sun et al., 2017) and offers enormous promise for tick research. However, these evolutionarily distinct hematophagous arthropods have quite different embryonic development and life histories than insects (Santos et al., 2013). Germline transformation methods that work for insects may require optimization for ticks. Recently, CRISPR-Cas was used to edit a mite, Tetranychus urticae, genome, the first for a chelicerate species and providing proofof-concept that CRISPR-Cas9 can be used to create gene knockouts in mites (Dermauw et al., 2020). Development of such methods in ticks that allow gene knockout, knock-in, and gene replacement, is urgently needed to facilitate an understanding of tick genetics, biochemistry, development, and behavior. Additionally, the development of effective and reliable ancillary methods such as identification of promoters, transformation markers, embryology, and embryo injection, are needed for effective germline transformation. Here, we are reviewing the use of CRISPR/Cas, the most promising geneediting approach, for tick genetic transformation and the ancillary methods needed for transgenic tick development.

\section{CURRENT RESEARCH AND FUTURE DEVELOPMENT TOWARDS TICK GENETIC MANIPULATION}

\section{Ancillary Methods for Transgenesis Transformation Markers}

Screening for mutants is a challenge for non-model organism transgenesis. In most arthropods there is an absence of mutant strains and corresponding rescue transgenes, as in the white-eye gene of Drosophila melanogaster (Rubin and Spradling, 1982). Instead, fluorescent markers such as enhanced green fluorescent protein (EGFP), are frequently used as reporters for gene insertion and expression (Berghammer et al., 1999; Horn et al., 2000; Horn et al., 2002; Thomas et al., 2002) and may be useful tools for tick transformation.

\section{3xP3-EGFP as a Universal Transformation Marker for Arthropod Transgenesis}

Evolutionarily conserved genetic circuitry governs all metazoan animal eye development under the control of the transcriptional activator Pax-6/Eyeless (Callaerts et al., 1997). Pax-6 homodimer binding site (P3) multimerization was shown to mediate photoreceptor-specific gene expression in D. melanogaster (Sheng et al., 1997). These studies led to the development of an artificial promoter, $3 \times \mathrm{x} 3.3 \mathrm{xP} 3$ has been employed to drive robust and eye-specific expression of EGFP (Berghammer et al., 1999; Horn et al., 2000) and identify transgenic individuals in several different insect orders. The artificial 3xP3 promoter construct mediates EGFP expression in the eyes of insects and, like the constitutive promoters mentioned below, can be used to identify transgenic organisms at all stages: larval, pupal, and adult (Horn et al., 2000). The evolutionary conserved function of Pax-6 in eye development of metazoans (Callaerts et al., 1997) suggests that the 3xP3-EGFP marker should apply to all eye-bearing animals. In mosquitoes, $3 \times \mathrm{P} 3$, expresses in eyes and ventral nerve cord (Volohonsky et al., 2017). An ortholog of D. melanogaster Pax-6 was also identified in the I. scapularis genome (ISCW003096/EEC03577) as well as other ticks such as Rhipicephalus sanguines (XM_037656705.1/XP_037512638) and Dermacentor silvarum (XM_037709500I/XP_037565428). It is therefore predicted that the $3 \mathrm{xP} 3$-EGFP marker should work in ticks that have well-developed eyes (such as Ambylomma, Dermacentor, and Rhipicephalus) and may have expression elsewhere in eyeless ticks of the genera Ixodes and Haemaphysalis (such as in the synganglion). Thus, $3 \mathrm{xP} 3$ with a tick-specific core promoter needs to be tested in ticks and new promoter/reporter systems need to be identified and developed. 


\section{Other Visible Markers}

A transformation marker system that results in phenotypes visible to the naked eye due to changes in the color of melanin pigments is highly desirable for tick transgenesis. The final step of enzymatically-regulated melanin biogenesis is the conversion of dopachrome into dihydroxyindoles, a reaction catalyzed by a class of enzymes called dopachrome tautomerases (DCT) and the functionally redundant enzyme in insects, bacteria, and fungi, dopachrome converting enzyme/yellow protein (DCE) (Rosani et al., 2019). Yellow protein marker has been effectively utilized in mosquito transgenics (Li et al., 2017a). We identified an ortholog of DCT in the I. scapularis genome (ISCW009232) that may have similar melanin biogenesis functions and needs to be tested. Unfortunately, not all markers from other arthropods may be suitable for ticks. For instance, overexpression or knockout of Arylalkylamine- $\mathrm{N}$-acetyl transferase and $\beta$-alanyldopamine synthetase (Bm-ebony) lightens coloration in Bombyx mori, Harmonia axyridis and D. melanogaster (Osanai-Futahashi et al., 2012), but their orthologs in I. scapularis genome have not been found. However, markers from more closely related arthropods may offer more promise. Mutations in a gene encoding a phytoene desaturase (tetur01g11270) were shown to cause an albino phenotype in T. utricae (lack of red pigment in the front legs and eyes) (Bryon et al., 2017). Knockout screening was enabled in this mite by injecting sgRNA and Cas9 into unfertilized females which produce only haploid male progeny that were easily screened for the albino phenotype (Dermauw et al., 2020). Although the orthologous sequences have not been identified in any tick genome, a similar gene product will be a promising target to develop for tick markers.

\section{Promoters}

Constitutive promoters allow the detection of transformants at all life stages. The polyubiquitin promoter was successfully utilized to generate the transformation marker PUbnlsEGFP in D. melanogaster (Handler and Harrell, 1999), and later to identify transformants in the Caribbean fruit fly, Anastrepha suspensa (Handler and Harrell, 2001), the Australian sheep blowfly, Lucilia cuprina (Heinrich et al., 2002), and mosquitoes (Anderson et al., 2010). Actin5C derived from D. melanogaster is another commonly used constitutive promoter to drive EGFP. The actin5C promoter works well at all developmental stages of D. melanogaster and the mosquitoes Aedes aegypti, Anopheles stephensi, and Culex quinquefasciatus (Catteruccia et al., 2000; (Pinkerton et al., 2000; Allen et al., 2001) but mediates only low and non-uniform EGFP expression levels in the stable fly, Stomoxys calcitrans (O'Brochta et al., 2000), suggesting that it is not broadly useful for all insects. Other promoters from $D$. melanogaster genes such as HSP70 and HSP82 have also been successfully used to drive marker genes in a variety of insects. While these promoters are widely useful, they are not universal. Transformation markers based on constitutive promoters have only been applied to closely related species, mostly within Diptera. Whether any such promoter can be functional across a wide range of insect orders, let alone more distantly related arthropods, remains to be tested.
Mammalian promoters with a GFP reporter, e.g. Cytomegalovirus immediate early promoter, human elongation factor la promoter, Human Ubiquitin C promoter, and chicken $\beta$ Actin promoter coupled with CMV early enhancer (CAGG), drive gene expression in Drosophila cell lines (Qin et al., 2010) and may be more broadly useful. Encouragingly, CAGG mCherry promoter was used to successfully transform an I. scapularis cell line, ISE6 (Oliver et al., 2015), yet it remains to be tested in tick embryos.

In order for work on tick transformation to proceed, endogenous tick promoters that work in a variety of tick species need to be identified. At the very least, a constitutive promoter that allows immediate identification of mutants after hatching is needed. Moving forward, tissue-specific or inducible promoters (such as the mosquito vitellogenin promoter that is fat body-specific and is activated after blood-feeding) would permit further refinement in tick transgenics. The availability of tick cell lines (Kurtti et al., 2008) provides an excellent tool for easily testing promoters in vitro before more challenging in vivo validation by embryo injections.

\section{Early Embryonic Development}

For the generation of successful stable mutants by embryo injection, early embryonic events such as the timing of cellularization and gonadal cell formation are essential to understand. This information is lacking in ticks and needs to be determined for efficient transgenic protocol development using CRISPR-Cas9 for knockout and knock-ins or classical transgenics using transposable elements.

Development of an embryo injection procedure requires knowledge of early embryonic development, including information about the timing of cellularization, anterior/posterior axis, and gonadal cell formation (pole cells in insects), but is currently lacking in ticks. For instance, only a handful of papers have observed embryogenesis in Ixodid (hard) ticks (Santos et al., 2013; Friesen et al., 2016), and none in the genus Ixodes. Even among these studies, early mitotic divisions were not examined because the earliest embryos used were $\sim 24 \mathrm{~h}$ post egg laying in both Rhipicephalus microplus (Santos et al., 2013) and Dermacentor andersoni (Friesen et al., 2016). At $24 \mathrm{~h}$ post egg laying, the embryos were already near the fifth mitotic division and the nuclei were located in the periphery of the egg (Santos et al., 2013; Friesen et al., 2016). Based on these early divisions, Friesen et al. (2016) suggested that if nuclear division occurs at a constant rate, the post-oviposition mitotic division rate in D. andersoni will be every $5 \mathrm{~h}$. This nuclear division rate is much slower than described in Drosophila melanogaster, where early mitotic divisions occur as fast as every $8 \mathrm{~min}$ (Foe and Alberts, 1983; Gilbert, 2000). In D. melanogaster embryos, the first 13 nuclear divisions occur without cytokinesis, resulting in the syncytial blastoderm. Whether or not the early mitotic divisions in tick embryos are holoblastic (with mitosis and cytokinesis) or syncytial remains to be confirmed (Fagotto et al., 1988; Campos et al., 2006; Santos et al., 2013; Friesen et al., 2016). Ixodes ticks have a longer embryogenesis time ( $\sim 40$ days compared to $\sim 11$ days of $R$. microplus) suggesting that the early embryonic events will be delayed in these ticks. Understanding these embryonic development events in ticks would facilitate efficient transgenesis by embryo injection. 
Strategies for Tick Gene-Editing

Genetic Manipulation by Injecting CRISPR/Cas9

Components in Embryos

Microinjection into newly deposited arthropod eggs (embryos) allows modification of the embryonic germline before it has differentiated (prior to cellularization), ensuring a heritable modification (Figure 1). Although approaches such as the gene gun (Thomas et al., 2001) and electroporation (Kamadar et al., 1992) have been tested in arthropods, embryo microinjection remains the most common approach for delivering gene-editing tools to the nucleus for genome modification. An embryo injection protocol was first established for the genetic transformation of $D$. melanogaster using transposable elements. This classical protocol (Rubin and Spradling, 1982) has been adapted to allow the injection of various types of nucleic acid constructs such as

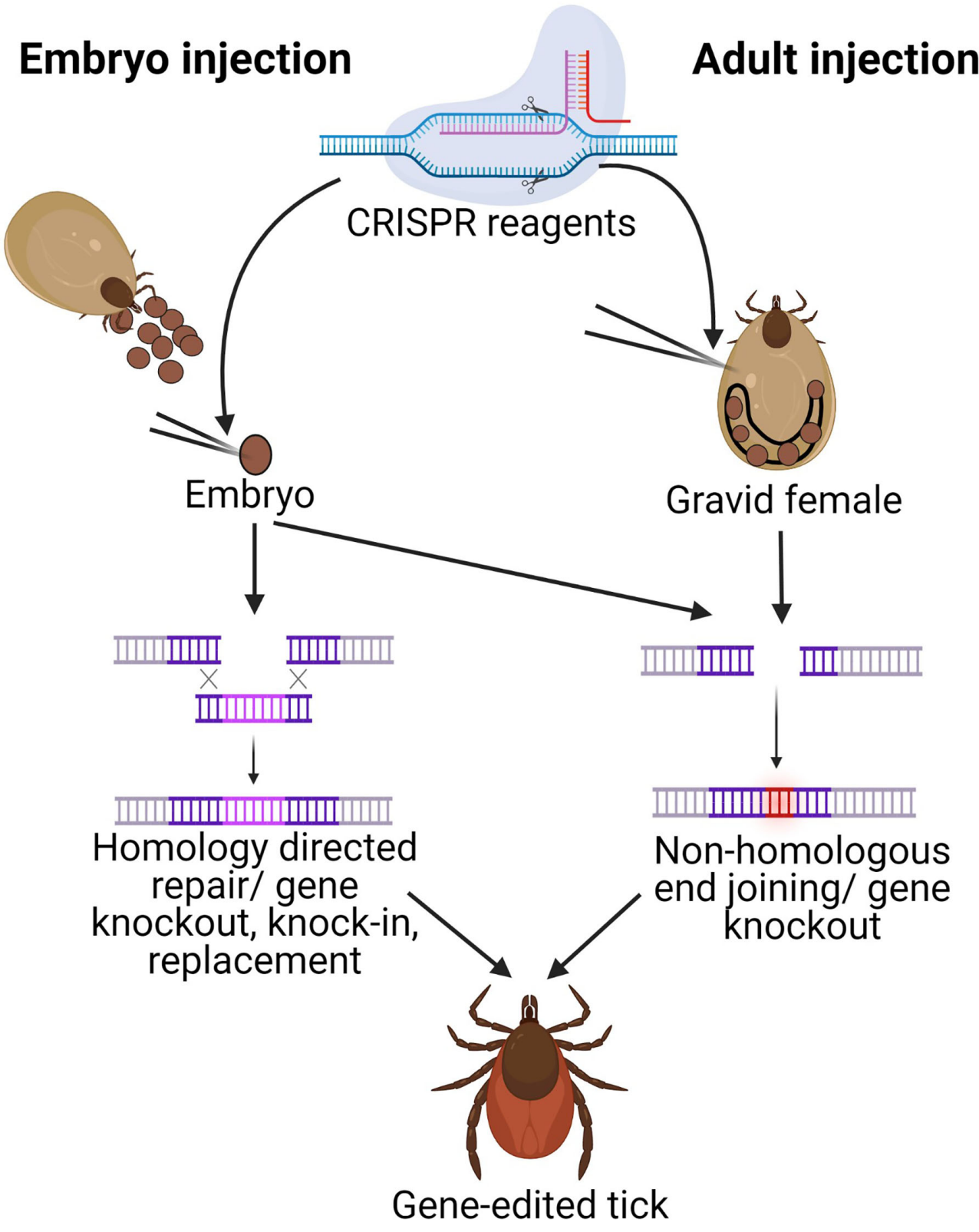

FIGURE 1 | Schematic of gene-editing in ticks by injecting either embryos or gravid adults. CRISPR reagents are injected either into newly deposited embryos (embryo injection) or into gravid females (adult injection). Adult injections require ReMOT components (ligand attached to Cas9 protein and endosomal escape reagent) in addition to sgRNA for delivery to the egg. Both techniques utilize Cas9 to induce a dsDNA break at the sgRNA site.The cell's repair machinery can introduce mutations through non-homologous end joining (NHEJ) in either technique for gene knockouts, but only embryo injections can currently introduce plasmids with homology arms necessary for homology directed repair (HDR) for gene knock-in. Image generated in Biorender.com. 
transformation vectors (P-elements, Piggybac, Hermes, etc.) and their helper plasmids, RNA, single guide RNA (sgRNA), plasmids, and Cas9 mRNA (or Cas9 protein).

The foundational work to develop injection methods has made the generation of transgenic Drosophila and even mosquitoes (Aedes and Anopheles) a routine laboratory procedure. Details about the physical operations involved in producing transformants such as DNA preparation, eggs, needles, and injections have been thoroughly described for these insects (Kiehart et al., 2007; Kistler et al., 2015). The general procedure involves collecting freshly laid eggs and dechorionating them using diluted bleach (Iwamatsu, 1983). The eggs are then attached to a glass coverslip with double-sided tape and slightly desiccated to make room for the injection volume. Preblastoderm stage eggs are then injected at their posterior end (before pole cell formation) with the injection mix. The resulting adults $\left(\mathrm{G}_{0}\right)$ are backcrossed and subsequent progeny are screened for the presence of the marker gene.

We recently developed an embryo injection protocol for $I$. scapularis and provided proof-of-principle for gene-editing in ticks. (Sharma et al., 2020, preprint). While early embryology in ticks is still not completely understood, our preliminary work has opened the door for further refinement of the injection protocol and resulting gene-editing methods. However, heritable germline mutations in ticks remain to be demonstrated. We expect that development of the above-mentioned ancillary methods coupled with our embryo injection protocol will make the generation of transgenic ticks a routine laboratory procedure, similar to mosquitoes.

\section{Genetic Manipulation by Injecting CRISPR/Cas9 Components in Gravid Females}

Despite our recent success at gene-editing ticks, tick embryo microinjection remains technically challenging. Two alternative methods that bypass the requirement for embryonic microinjection have been developed for insects: 1. Receptor-Mediated Ovary Transduction of Cargo (ReMOT) and 2) Branched Amphiphilic Peptide Capsules (BAPC)- assisted CRISPR delivery (ChaverraRodriguez et al., 2018; Hunter et al., 2018) (Figure 1). ReMOT is based on delivery of the CRISPR/Cas9 ribonucleoprotein (RNP) complex (Cas9 with an sgRNA) by using peptide ligands derived from D. melanogaster yolk protein precursors (YPPs) fused to the Cas9-RNP complex. Chemical compounds such as chloroquine or saponin are used as endosomal escape reagents (EER) that facilitate the escape of the YPP-RNP complex from endosomes into the oocyte cytoplasm. The injection of the YPP-RNP/EER complex into the hemolymph of vitellogenic females of several insect species enabled targeted gene editing in embryos (Chaverra-Rodriguez et al., 2018; Heu et al., 2020; Macias et al., 2020; Shirai and Daimon, 2020). The BAPC-assisted CRISPR delivery involves the use of branched amphiphilic peptide capsules BAPtofect ${ }^{\mathrm{TM}}$ (Phoreus $^{\mathrm{TM}}$ Biotechnology, Inc. Olathe, Kansas, US) for delivery of CRISPR RNP into the ovary (Sukthankar et al., 2014) and was recently used to improve the delivery of CRISPR components into ovaries of the adult Asian citrus psyllid, Diaphorina citri (Hunter et al., 2018) to facilitate heritable gene editing.

Our recent work successfully utilized ReMOT technology for gene editing in I. scapularis suggesting promise for this technique as a straightforward method for gene knockout in ticks (Sharma et al., 2020, preprint). The ReMOT reagents were stable in ticks for a maximum of $48 \mathrm{~h}$, necessitating multiple injections during the relatively long egg maturation and deposition time in ticks. BAPC-assisted CRISPR delivery has yet to be tested in ticks, but this method relies on stable peptide capsules which may have improved stability and avoid the need for multiple hemolymph injections. However, a limitation of both of these alternate strategies is that they cannot currently be used for gene knockin (overexpression or replacement) as there is no mechanism for carrying template into the embryo for homology-directed repair, making them suitable only for gene knockout studies.

\section{DISCUSSION}

The availability of tick genome sequences (Cramaro et al., 2015; Gulia-Nuss et al., 2016; Cramaro et al., 2017; Nuss et al., 2018; Jia et al., 2020) have already made application of molecular methods possible in ticks. However, study of the molecular biology of ticks is currently limited by the applicability of genetic tools. The need to achieve efficient methods for germline transformation in ticks remains a high priority for functional genomics research. For this to happen, the groundwork needs to be built for ancillary methods such as embryology, embryo injection, and identification of tick promoters and markers. Embryo injection methods are crucial for the development of transgenic lines and necessary for other applications such as stable infection with symbionts. Similarly, the development of gene-editing methods that do not require embryo injection (such as ReMOT) can be used for gene knockouts to help make genetically engineered ticks a common lab practice. Additional refinement of this method may permit gene knock-ins to be used for different applications such as gene replacement and over-expression.

CRISPR/Cas technologies have made targeted genetic engineering feasible in most organisms. Proof-of-principle experiments in Ixodes demonstrate that this technique is feasible in ticks as well (Sharma et al., 2020 preprint). However, stable germline transformation in ticks has not yet been confirmed. Therefore, a future focus should be on creating stable lines for fundamental research. Beyond a fundamental understanding of tick biology, a further application of tick genetic transformation is population control by inserting gene drives. Gene drives, which bias inheritance towards a natural or synthetic genetic element or specific allele and lead to a preferential increase of a specific phenotype throughout a population (Alphey et al., 2020), are being developed for mosquito control. Several different gene drives, autonomous/ non-autonomous, split, self-limiting drives, etc., have been developed (Gantz et al., 2015; Hammond et al., 2016; Patil et al., 2018; Adolfi et al., 2020; Carballar-Lejarazú et al., 2020; Li et al., 2020). For instance, a male dominant allele to produce a single sex to reduce tick populations, or a trait to increase refractoriness to pathogens, could be effective strategies for managing tick-borne diseases. 
Further research into the areas identified in this opinion piece will provide the necessary steps to develop routine genetic transformations in ticks. Once this is possible, it will provide a badly needed set of tools to understand gene function in ticks. This will open the door to understanding a group of truly unique arthropods and how we might manage these major vectors of human and animal disease.

\section{DATA AVAILABILITY STATEMENT}

The original contributions presented in the study are included in the article/supplementary material. Further inquiries can be directed to the corresponding author.

\section{REFERENCES}

Adolfi, A., Gantz, V. M., Jasinskiene, N., Lee, H. F., Hwang, K., Terradas, G., et al. (2020). Efficient Population Modification Gene-Drive Rescue System in the Malaria Mosquito Anopheles Stephensi. Nat. Commun. 11, 1-13. doi: 10.1038/ s41467-020-19426-0

Allen, M. L., O’Brochta, D. A., Atkinson, P. W., and Levesque, C. S. (2001). Stable, Germ-Line Transformation of Culex Quinquefasciatus (Diptera: Culicidae). J. Med. Entomol. 38, 701-710. doi: 10.1603/0022-2585-38.5.701

Alphey, L. S., Crisanti, A., Randazzo, F., and Akbari, O. S. (2020). Opinion: Standardizing the Definition of Gene Drive. Proc. Natl. Acad. Sci. U. S. A. 117, 30864-30867. doi: 10.1073/pnas.2020417117

Anderson, M. A. E., Gross, T. L., Myles, K. M., and Adelman, Z. N. (2010). Validation of Novel Promoter Sequences Derived From Two Endogenous Ubiquitin Genes in Transgenic Aedes Aegypti. Insect Mol. Biol. 19, 441-449. doi: 10.1111/j.1365-2583.2010.01005.x

Bassett, A. R., and Liu, J. L. (2014). CRISPR/Cas9 and Genome Editing in Drosophila. J. Genet. Genomics 41, 7-19. doi: 10.1016/j.jgg.2013.12.004

Berghammer, A. J., Klingler, M., and Wimmer, E. A. (1999). A Universal Marker for Transgenic Insects. Nature 402, 370-371. doi: 10.1038/46463

Bryon, A., Kurlovs, A. H., Dermauw, W., Greenhalgh, R., Riga, M., Grbić, M., et al. (2017). Disruption of a Horizontally Transferred Phytoene Desaturase Abolishes Carotenoid Accumulation and Diapause In Tetranychus Urticae. Proc. Natl. Acad. Sci. U. S. A. 114 (29), E5871-E5880. doi: 10.1073/pnas.1706865114

Callaerts, P., Halder, G., and Gehring, W. J. (1997). Pax-6 in Development and Evolution. Annu. Rev. Neurosci. 20, 483-532. doi: 10.1146/annurev.neuro.20.1.483

Campos, E., Moraes, J., Façanha, A. R., Moreira, É., Valle, D., Abreu, L., et al. (2006). Kinetics of Energy Source Utilization in Boophilus Microplus (Canestrini 1887) (Acari: Ixodidae) Embryonic Development. Vet. Parasitol. 138, 349-357. doi: 10.1016/j.vetpar.2006.02.004

Carballar-Lejarazú, R., Ogaugwu, C., Tushar, T., Kelsey, A., Pham, T. B., Murphy, J., et al. (2020). Next-Generation Gene Drive for Population Modification of the Malaria Vector Mosquito, Anopheles Gambiae. Proc. Natl. Acad. Sci. U. S. A. 117, 22805-22814. doi: 10.1073/pnas.2010214117

Catteruccia, F., Nolan, T., Loukeris, T. G., Blass, C., Savakis, C., Kafatos, F. C., et al. (2000). Stable Germline Transformation of the Malaria Mosquito Anopheles Stephensi. Nature 405, 959-962. doi: 10.1038/35016096

Chaverra-Rodriguez, D., Macias, V. M., Hughes, G. L., Pujhari, S., Suzuki, Y., Peterson, D. R., et al. (2018). Targeted Delivery of CRISPR-Cas9 Ribonucleoprotein Into Arthropod Ovaries for Heritable Germline Gene Editing. Nat. Commun. 9, 1-11. doi: 10.1038/s41467-018-05425-9

Commins, S. P., and Platts-Mills, T. A. E. (2013). Tick Bites and Red Meat Allergy. Curr. Opin. Allergy Clin. Immunol. 13, 354-359. doi: 10.1097/ACI.0b013e3283624560

Cramaro, W. J., Revets, D, Hunewald, O. E., Sinner, R., Reye, A. L., Muller, C. P., et al. (2015). Integration of Ixodes Ricinus Genome Sequencing With Transcriptome and Proteome Annotation of the Naïve Midgut. BMC Genomics 16, 871. doi: 10.1186/s12864-015-1981-7

Cramaro, W. J., Hunewald, O. E., Bell-Sakyi, L., and Muller, C. P. (2017). Genome Scaffolding and Annotation for the Pathogen Vector Ixodes Ricinus by Ultra-

\section{AUTHOR CONTRIBUTIONS}

AN, AS, and MG-N wrote the initial drafts. MG-N and AN wrote the final draft. All authors contributed to the article and approved the submitted version.

\section{FUNDING}

This work was funded by NIH-NIAID R21AI128393 and Plymouth Hill Foundation, NY to MG-N, Startup funds from the University of Nevada to AN, Peer-to-Peer Grant from IGTRCN to AS.

Long Single Molecule Sequencing. Parasit. Vectors 10 (1), 71. doi: 10.1186/ s13071-017-2008-9

Dermauw, W., Jonckheere, W., Riga, M., Livadaras, I., Vontas, J., and Van Leeuwen, T. (2020). Targeted Mutagenesis Using CRISPR-Cas9 in the Chelicerate Herbivore Tetranychus Urticae. Insect Biochem. Mol. Biol. 120, 103347. doi: 10.1016/j.ibmb.2020.103347

Eisen, R. J., and Eisen, L. (2018). The Blacklegged Tick, Ixodes Scapularis: An Increasing Public Health Concern. Trends Parasitol. 34, 295-309. doi: 10.1016/ j.pt.2017.12.006

Fagotto, F., Hess, E., and Aeschlimann, A. (1988). The Early Embryonic Development of the Argasid Tick Ornithodorus Moubata (Acarina: Ixodoidea: Argasidae). Entomol. Gen. 13, 1-8. doi: 10.1127/entom.gen/13/1988/1

Foe, V. E., and Alberts, B. M. (1983). Studies of Nuclear and Cytoplasmic Behavior During the Five Mitotic Cycles That Precede Gastrulation in Drosophila Embryogenesis. J. Cell Sci. 61, 31-70.

Friesen, K. J., Dixon, M., and Lysyk, T. J. (2016). Embryo Development and Morphology of the Rocky Mountain Wood Tick (Acari: Ixodidae). J. Med. Entomol. 53, 279-289. doi: 10.1093/jme/tjv193

Gantz, V. M., Jasinskiene, N., Tatarenkova, O., Fazekas, A., Macias, V. M., Bier, E., et al. (2015). Highly Efficient Cas9-mediated Gene Drive for Population Modification of the Malaria Vector Mosquito Anopheles Stephensi. Proc. Natl. Acad. Sci. U. S. A. 112, E6736-E6743. doi: 10.1073/pnas.1521077112

Gilbert, S. F. (2000). "An Introduction to Early Developmental Processes," in Developmental Biology, 6th edition (Sunderland, MA: Sinauer Associates).

Gilles, A. F., Schinko, J. B., and Averof, M. (2015). Efficient CRISPR-mediated Gene Targeting and Transgene Replacement in the Beetle Tribolium Castaneum. Development 142, 2832-2839. doi: 10.1242/dev.125054

Gulia-Nuss, M., Nuss, A. B., Meyer, J. M., Sonenshine, D. E., Roe, R. M., Waterhouse, R. M., et al. (2016). Genomic Insights Into the Ixodes Scapularis Tick Vector of Lyme Disease. Nat. Commun. 7, 10507. doi: 10.1038/ncomms10507

Hammond, A., Galizi, R., Kyrou, K., Simoni, A., Siniscalchi, C., Katsanos, D., et al. (2016). A CRISPR-Cas9 Gene Drive System Targeting Female Reproduction in the Malaria Mosquito Vector Anopheles Gambiae. Nat. Biotechnol. 34, 78-83. doi: 10.1038/nbt.3439

Handler, A. M., and Harrell, R. A. (1999). Germline Transformation of Drosophila Melanogaster With the piggyBac Transposon Vector. Insect Mol. Biol. 8, 449457. doi: 10.1046/j.1365-2583.1999.00139.x

Handler, A. M., and Harrell, R. A. (2001). Transformation of the Caribbean Fruit Fly, Anastrepha Suspensa, With a piggyBac Vector Marked With Polyubiquitin-Regulated GFP. Insect Biochem. Mol. Biol. 31, 199-205. doi: 10.1016/S0965-1748(00)00119-3

Heinrich, J. C., Li, X., Henry, R. A., Haack, N., Stringfellow, L., Heath, A. C. G., et al. (2002). Germ-Line Transformation of the Australian Sheep Blowfly Lucilia Cuprina. Insect Mol. Biol. 11, 1-10. doi: 10.1046/j.0962-1075.2001.00301.x

Heu, C. C., McCullough, F. M., Luan, J., and Rasgon, J. L. (2020). CRISPR-Cas9Based Genome Editing in the Silverleaf Whitefly ( Bemisia Tabaci ). CRISPR J. 3, 89-96. doi: 10.1089/crispr.2019.0067

Horn, C., Jaunich, B., and Wimmer, E. A. (2000). Highly Sensitive, Fluorescent Transformation Marker for Drosophila Transgenesis. Dev. Genes Evol. 210, 623-629. doi: 10.1007/s004270000111 
Horn, C., Schmid, B. G. M., Pogoda, F. S., and Wimmer, E. A. (2002). Fluorescent Transformation Markers for Insect Transgenesis. Insect Biochem. Mol. Biol. 32, 1221-1235. doi: 10.1016/S0965-1748(02)00085-1

Hunter, W. B., Gonzalez, M. T., and Tomich, J. (2018). BAPC-Assisted CRISPR/ Cas9 System: Targeted Delivery Into Adult Ovaries for Heritable Germline Gene Editing (Arthropoda: Hemiptera). bioRxiv 478743. doi: 10.1101/478743

Iwamatsu, T. (1983). A New Technique for Dechorionation and Observations on the Development of the Naked Egg in Oryzias Latipes. J. Exp. Zool. 228, 83-89. doi: 10.1002/jez.1402280109

Jia, N., Wang, J., Shi, W., Du, L., Sun, Y., Zhan, W., et al. (2020). Tick Genome and Microbiome Consortium (Tigmic), Zhao F, Cao Wc. Large-Scale Comparative Analyses of Tick Genomes Elucidate Their Genetic Diversity and Vector Capacities. Cell 182 (5), 1328-1340.e13. doi: 10.1016/j.cell.2020.07.023

Jongejan, F., and Uilenberg, G. (2004). The Global Importance of Ticks. Parasitology 129, Suppl:S3-14. doi: 10.1017/S0031182004005967

Kamadar, P., Von Allmen, G., and Victoria, F. (1992). Transient Expression of DNA in Drosophila Via Electroporation. Nucleic Acids Res. 20, 3526. doi: $10.1093 /$ nar/20.13.3526

Karim, S., Kenny, B., Troiano, E., and Mather, T. N. (2008). RNAi-Mediated Gene Silencing in Tick Synganglia: A Proof of Concept Study. BMC Biotechnol. 8, 30. doi: 10.1186/1472-6750-8-30

Kiehart, D. P., Crawford, J. M., and Montague, R. A. (2007). Collection, Dechorionation, and Preparation of Drosophila Embryos for Quantitative Microinjection. Cold Spring Harb. Protoc. 2007, pdb.prot4717-pdb.prot4717. doi: $10.1101 /$ pdb.prot 4717

Kistler, K. E., Vosshall, L. B., and Matthews, B. J. (2015). Genome Engineering With CRISPR-Cas9 in the Mosquito Aedes Aegypti. Cell Rep. 11, 51-60. doi: 10.1016/j.celrep.2015.03.009

Kohno, H., Suenami, S., Takeuchi, H., Sasaki, T., and Kubo, T. (2016). Production of Knockout Mutants by CRISPR/Cas9 in the European Honeybee, Apis Mellifera L. Zoolog. Sci. 33, 505-512. doi: 10.2108/zs160043

Kurtti, T. J., Mattila, J. T., Herron, M. J., Felsheim, R. F., Baldridge, G. D., Burkhardt, N. Y., et al. (2008). Transgene Expression and Silencing in a Tick Cell Line: A Model System for Functional Tick Genomics. Insect Biochem. Mol. Biol. 38, 963-968. doi: 10.1016/j.ibmb.2008.07.008

Li, M., Au, L. Y. C., Douglah, D., Chong, A., White, B. J., Ferree, P. M., et al. (2017b). Generation of Heritable Germline Mutations in the Jewel Wasp Nasonia Vitripennis Using CRISPR/Cas9. Sci. Rep. 7, 1-7. doi: 10.1038/s41598-017-00990-3

Li, M., Bui, M., Yang, T., Bowman, C. S., White, B. J., and Akbari, O. S. (2017a). Germline Cas9 Expression Yields Highly Efficient Genome Engineering in a Major Worldwide Disease Vector, Aedes Aegypti. Proc. Natl. Acad. Sci. U. S. A. 114 (49), E10540-E10549. doi: 10.1073/pnas.1711538114

Li, M., Yang, T., Kandul, N. P., Bui, M., Gamez, S., Raban, R., et al. (2020). Development of a Confinable Gene Drive System in the Human Disease Vector Aedes Aegypti. Elife 9, e51701. doi: 10.7554/eLife.51701

Ma, S., Chang, J., Wang, X., Liu, Y., Zhang, J., Lu, W., et al. (2014). CRISPR/Cas9 Mediated Multiplex Genome Editing and Heritable Mutagenesis of BmKu70 in Bombyx Mori. Sci. Rep. 4, 1-6. doi: 10.1038/srep04489

Macias, V. M., McKeand, S., Chaverra-Rodriguez, D., Hughes, G. L., Fazekas, A., Pujhari, S., et al. (2020). Cas9-Mediated Gene-Editing in the Malaria Mosquito Anopheles Stephensi by ReMOT Control. G3 Genes Genomes Genet. 10, g3.401133.2020. doi: 10.1534/g3.120.401133

Nuss, A. B., Sharma, A., and Gulia-Nuss, M. (2018). Chicago and Dovetail Hi-C Proximity Ligation Yield Chromosome Length Scaffolds Of Ixodes Scapularis Genome. BioRxiv, 392126. doi: 10.1101/392126

O'Brochta, D. A., Atkinson, P. W., and Lehane, M. J. (2000). Transformation of Stomoxys Calcitrans With a Hermes Gene Vector. Insect Mol. Biol. 9, 531-538. doi: 10.1046/j.1365-2583.2000.00217.x

Oliver, J. D., Chávez, A. S. O., Felsheim, R. F., Kurtti, T. J., and Munderloh, U. G. (2015). An Ixodes Scapularis Cell Line With a Predominantly Neuron-Like Phenotype. Exp. Appl. Acarol. 66, 427-442. doi: 10.1007/s10493-015-9908-1

Osanai-Futahashi, M., Ohde, T., Hirata, J., Uchino, K., Futahashi, R., Tamura, T., et al. (2012). A Visible Dominant Marker for Insect Transgenesis. Nat. Commun. 3, 1295. doi: 10.1038/ncomms2312

Patil, P. B., Gorman, K. J., Dasgupta, S. K., Reddy, K. V. S., Barwale, S. R., and Zehr, U. B. (2018). Self-Limiting OX513A Aedes Aegypti Demonstrate Full Susceptibility to Currently Used Insecticidal Chemistries as Compared to Indian Wild-Type Aedes Aegypti. Psyche J. Entomol. 2018, 7. doi: 10.1155/2018/7814643
Pienaar, R., Neitz, A., and Mans, B. J. (2018). Tick Paralysis: Solving an Enigma. Vet. Sci. 5 (2):53. doi: 10.3390/vetsci5020053

Pinkerton, A. C., Michel, K., O’Brochta, D. A., and Atkinson, P. W. (2000). Green Fluorescent Protein as a Genetic Marker in Transgenic Aedes Aegypti. Insect Mol. Biol. 9, 1-10. doi: 10.1046/j.1365-2583.2000.00133.x

Qin, J. Y., Zhang, L., Clift, K. L., Hulur, I., and Xiang, A. P. (2010). Systematic Comparison of Constitutive Promoters and the Doxycycline-Inducible Promoter. PloS One 5, 10611. doi: 10.1371/journal.pone.0010611

Rochlin, I., and Toledo, A. (2020). Emerging Tick-Borne Pathogens of Public Health Importance: A Mini-Review. J. Med. Microbiol. 69, 781-791. doi: $10.1099 / j m m .0 .001206$

Rosani, U., Domeneghetti, S., Maso, L., Wegner, K. M., and Venier, P. (2019). An Evolutionary Perspective of Dopachrome Tautomerase Enzymes in Metazoans. Genes 10 (7), 495. doi: 10.3390/genes 10070495

Rubin, G. M., and Spradling, A. C. (1982). Genetic Transformation of Drosophila With Transposable Element Vectors. Science (80-.) 218, 348-353. doi: 10.1126/ science. 6289436

Santos, V. T., Ribeiro, L., Fraga, A., de Barros, C. M., Campos, E., Moraes, J., et al. (2013). The Embryogenesis of the Tick Rhipicephalus (Boophilus) Microplus: The Establishment of a New Chelicerate Model System. Genesis 51, 803-818. doi: 10.1002/dvg.22717

Sharma, A., Pham, M. N., Reyes, J. B., Chana, R., Yim, W. C., Heu, C. C., et al. (2020). Cas9-Mediated Gene-Editing in the Black-Legged Tick, Ixodes Scapularis, by Embryo Injection and ReMOT Control. SSRN Electron. J. doi: $10.2139 /$ ssrn.3691041

Sheng, G., Thouvenot, E., Schmucker, D., Wilson, D. S., and Desplan, C. (1997). Direct Regulation of Rhodopsin 1 by Pax-6/eyeless in Drosophila: Evidence for a Conserved Function in Photoreceptors. Genes Dev. 11, 1122-1131. doi: 10.1101/gad.11.9.1122

Shirai, Y., and Daimon, T. (2020). Mutations in Cardinal are Responsible for the Red-1 and Peach Eye Color Mutants of the Red Flour Beetle Tribolium Castaneum. Biochem. Biophys. Res. Commun. 529, 372-378. doi: 10.1016/ j.bbrc.2020.05.214

Steinke, J. W., Platts-Mills, T. A., and Commins, S. P. (2015). The Alpha-Gal Story: Lessons Learned From Connecting the Dots. J. Allergy Clin. Immunol. 135 (3), 589-597. doi: 10.1016/j.jaci.2014.12.1947

Sukthankar, P., Avila, L. A., Whitaker, S. K., Iwamoto, T., Morgenstern, A., Apostolidis, C., et al. (2014). Branched Amphiphilic Peptide Capsules: Cellular Uptake and Retention of Encapsulated Solutes. Biochim. Biophys. Acta - Biomembr. 1838, 2296-2305. doi: 10.1016/ j.bbamem.2014.02.005

Sun, D., Guo, Z., Liu, Y., and Zhang, Y. (2017). Progress and Prospects of CRISPR/ Cas Systems in Insects and Other Arthropods. Front. Physiol. 8, 608 doi: 10.3389/fphys.2017.00608

Thomas, J. L., Bardou, J., L'hoste, S., Mauchamp, B., and Chavancy, G. (2001). A Helium Burst Biolistic Device Adapted to Penetrate Fragile Insect Tissues. J. Insect Sci. 1, 1-10. doi: 10.1673/031.001.0901

Thomas, J. L., Da Rocha, M., Besse, A., Mauchamp, B., and Chavancy, G. (2002). $3 \times$ P3-EGFP Marker Facilitates Screening for Transgenic Silkworm Bombyx Mori L. From the Embryonic Stage Onwards. Insect Biochem. Mol. Biol. 32, 247-253. doi: 10.1016/S0965-1748(01)00150-3

Tick-borne Disease Working Group. 2018 Report to Congress. Available at: https:// www.hhs.gov/sites/default/files/tbdwg-report-to-congress-2018.pdf.

Volohonsky, G., Hopp, A. K., Saenger, M., Soichot, J., Scholze, H., Boch, J., et al. (2017). Transgenic Expression of the Anti-parasitic Factor TEP1 in the Malaria Mosquito Anopheles Gambiae. PloS Pathog. 13 (1), e1006113. doi: 10.1371/ journal.ppat.1006113

Conflict of Interest: The authors declare that the research was conducted in the absence of any commercial or financial relationships that could be construed as a potential conflict of interest.

Copyright (c) 2021 Nuss, Sharma and Gulia-Nuss. This is an open-access article distributed under the terms of the Creative Commons Attribution License (CC BY). The use, distribution or reproduction in other forums is permitted, provided the original author(s) and the copyright owner(s) are credited and that the original publication in this journal is cited, in accordance with accepted academic practice. No use, distribution or reproduction is permitted which does not comply with these terms. 